organized labour and companies in the British political crisis of 1973-1974 may simply be a correlate of an unprecedented period of economic growth and affluence. More importantly, the logic of pressure group politics would seem to suggest that a "centripetal solution" may be an expedient rather than a cure. Organized power groups of the kind referred to in the book are highly opportunistic units likely to adapt operationally to political change. Can we really be certain, in other words, that corporate forces will ultimately be less effective at a greater political level than the author contends they are now at the national? Alternatively, could not one reverse Professor Ionescu's conclusion and conjecture with equal reason that constitutional devolution may provide a political structure less amenable to manipulation by corporate forces?

Whether or not one agrees with the author, it is clear that his book makes a major contribution to the on-going debate on power in modern society. For anyone interested in that question or the related one of European unity, this book should be required reading.

Calvin A. Woodward

\title{
GotTfried-Karl KindermanN
}

Pekings chinesische Gegenspieler. Theorie und Praxis nationalchinesischen Widerstandes auf Taiwan

Droste Verlag Düsseldorf, 1977, 290 S., 34,- DM

Am 12. Januar 1977 gab der Außenminister von Barbados den Abbruch der diplomatischen Beziehungen seines Staates mit der Republik China bekannt, zur Begründung auf die „internationalen Realitäten “ verweisend1. Daß die Realität, was das geteilte China anlangt, eine ganz andere sei, als hier zum Ausdruck kommt, sucht Kindermann mit dem vorliegenden Buch nachzuweisen und ins Bewußtsein der deutschsprachigen Offentlichkeit zu rücken: Unabhängig von völkerrechtlicher Beurteilung ${ }^{2}$ müsse - ähnlich der Lage im geteilten Deutschland, die zur Ostpolitik der Brandt/Scheel-Regierung führte - die "Realität“ Taiwan in die außenpolitische Rechnung einbezogen werden (Vorwort, S. 12). Der Münchner Politikwissenschaftler könnte dabei auch auf Tendenzen im sozialistischen Lager verweisen, spricht doch die Sowjetunion gelegentlich korrekt von der „Republik China" und hat sie bereits bekundet, sich die Teilnahme Taiwans an den Olympischen Spielen in Moskau vorstellen zu können. Aus volksrepublikanisch-chinesischer Sicht freilich wird das Unterfangen Kindermanns, wie jede Befassung mit Taiwan, die „zwei China“, „ein China, ein Taiwan“, „ein China, zwei Regierungen“ oder gar ein Recht Taiwans auf Unabhängigkeit für möglich hält, als Einmischung in chinesische Angelegenheiten erscheinen ${ }^{3}$.

In seinem neuen Taiwan-Buch ${ }^{4}$ beschreibt Kindermann die Grundlagen des Selbstverständnisses der Chiang-Administration sowie die innen- und (knapp) die außenpolitische Lage. Ein ausführliches Kapitel ist der agrarpolitischen Entwicklung

\footnotetext{
1 Archiv der Gegenwart 20713 A.

2 Dazu z. B. G. Kaminski, China-Taiwan, 1973; s. auch F. Münch, Archiv des Völkerrechts 14 (1969/70), S. $295 \mathrm{f}$.

3 In diesem Sinne die Außerungen des ZK-Mitgliedes Liao Tscheng-dschi in seiner Rede zum 30. Jahrestag des Volksaufstandes vom 28. Februar 1947 (dazu Kindermann, S. 51), vgl. "Peking-Rundschau“ 1977, Heft 10, S. 6.

41974 gab er den Band ,Stimmen des Anderen China - Nationalchinesische Experten zu entwicklungspolitischen Alternativen für China ${ }^{\alpha}$ heraus.
} 
gewidmet. Durchmischt sind diese darstellenden Teile mit teils bekannten, teils schwer zugänglichen, teils erstmals veröffentlichten Dokumenten: Texte der die ideologische Debatte prägenden Autoren Sun Yat-sen, Chiang Kai-shek, Hu Shih, Sun Yun-suan, Chiang Ching-kuo, eigene Interviews mit dem Ministerpräsidenten und Regierungsbeamten, eine ausführliche Zeittafel zu Vorgeschichte und Geschichte des chinesischen Bürgerkrieges unter besonderer Berücksichtigung Taiwan betreffender Daten, schließlich ein Schriften- und ein Periodika-Verzeichnis. In den darstellenden Abschnitten, vor allem bei der Darstellung agrarpolitischer Pläne und Praxis konterkariert der Autor das taiwanesische Modell mit Hinweisen auf den Stand der Agrarrevolution auf dem Festland. Die abgedruckten Texte sind repräsentativ, wenngleich die Auszüge aus den Schriften des Generalissimus selbst vielleicht hätten ergänzt werden sollen mit Abschnitten aus dem programmatischen Werk "Chinas Destiny"5, das als „Mein Kampf" Chinas bezeichnet worden ist $^{6}$ und im Vergleich mit den programmatischen Schriften Maos aus der gleichen Phase $^{7}$ wertvolle Aufschlüsse liefert über die beiderseitigen Konzepte am Beginn des chinesischen Scheideweges. Der positive Gesamteindruck, den die von Kindermann präsentierten Texte auf den unbefangenen westlichen Leser machen werden, wäre allerdings bei ausführlichen Zitaten aus dem genannten Werk gefährdet ...

Das Buch ist insgesamt weniger polemisch angelegt, als dies sein Untertitel assoziieren lassen könnte; in einer leicht verständlichen, erkennbar an einen breiteren Interessentenkreis gerichteten Sprache verfaßt, wird es jedem nützen können, der sich über die taiwanesische Gegenwart und ihre Vorgeschichte unterrichten möchte. Nachgetragen sei - Kindermann hebt zu Recht die Abhängigkeit des weiteren Schicksals der Erben Chiang Kai-sheks von der nach Maos Tod noch schwerer prognostizierbaren Entwicklung in der Volksrepublik hervor (S. 85) -, daß mittlerweile auch der neue Parteichef Hua Kuo-feng seine Entschlossenheit betont hat, "die geheiligte Sache der Befreiung Taiwans und der Wiedervereinigung des Vaterlandes $\mathrm{zu}$ vollenden ${ }^{8 “}$, denn die "Landsleute in der Provinz Taiwan sind von unserem Fleisch und Blut ${ }^{9}$ “.

Philip Kunig

\section{WinfRIEd MANig}

\section{Steuern und Agrarentwicklung in Entwicklungsländern}

(Occasional Papers Nr. 5. Materialien zur Reihe Sozialökonomische Schriften zur Agrarentwicklung) Verlag der ssip-Schriften, Saarbrücken 1976, 213 S., DM 7,-.

Die Arbeit ist aus einer Vorlesung des Verfassers über „Besteuerung der Landwirtschaft in Entwicklungsländern" an der Universität Göttingen hervorgegangen. Sie behandelt den üblichen Kanon der allgemeinen und speziellen Steuerlehre. Beispiele für die Ausgestaltung einzelner Steuern und wirtschaftspolitische Anwendungen der Steuerwirkungs- und Inzidenzlehre beziehen sich aber vornehmlich auf den Agrarsektor in den Entwicklungsländern. Zusammenhänge zwischen Ausgabenund Einnahmenseite des Staatsbudgets und.Verflechtungen des Agrarsektors mit

\footnotetext{
5 Erstausgabe Chungking 1943; englisch New York 1947 und Taipei 1953.

6 Von dem Amerikaner Philip Jaffe.

7 Vor allem "Ưber die Neue Demokratie*, 1940; „Uber die Koalitionsregierung, 1945.

8 In seiner programmatischen Rede auf das Jahr 1977, vgl. „Peking-Rundschau“ 1977, Heft 1, S. 30 ff., 45.

9 Hongqi, Neujahr 1977.
} 